\title{
Frequent EGFR mutations in nonsmall cell lung cancer presenting with miliary intrapulmonary carcinomatosis
}

\author{
Shang-Gin Wu*, Fu-Chang Hü, Yih-Leong Chang", Yung-Chie Lee ${ }^{+}$, Chong-Jen Yu ${ }^{\S, f}$, \\ Yeun-Chung Chang ${ }^{f * *}$, Jenn-Yu Wu*, Jin-Yuan Shih ${ }^{\S, f}$ and Pan-Chyr Yang ${ }^{\S, f}$
}

ABSTRACT: Nonsmall cell lung cancer (NSCLC) presenting with miliary intrapulmonary carcinomatosis (MIPC) is rare. We investigated the clinical characteristics and epidermal growth factor receptor (EGFR) mutation rate of NSCLC patients with MIPC at initial diagnosis.

From June 2004 to December 2008, we screened newly diagnosed NSCLC patients for MIPC using image-based criteria. We recorded clinical data and analysed EGFR mutation status. For comparison, we collected specimens from stage IV NSCLC patients without MIPC tested for EGFR mutations from April 2001 to November 2008.

From 3,612 NSCLC patients, 85 patients with MIPC at initial diagnosis were identified; 81 had adenocarcinoma. Of the 85 patients, 60 had specimen sequencing to detect EGFR mutation; 42 (70\%) were positive. Compared with 673 stage IV patients without MIPC, patients with MIPC had higher EGFR mutation rate $(p=0.036)$; even male smokers had a high EGFR mutation rate $(91 \%)$. Multivariate analysis of prognostic factors for overall survival of the 85 patients with MIPC revealed that adenocarcinoma, absence of extrapulmonary metastasis and having EGFR mutation were associated with longer overall survival.

NSCLC patients with MIPC at initial diagnosis had higher rates of adenocarcinoma and EGFR mutation. EGFR tyrosine kinase inhibition may be the treatment of choice for NSCLC patients with MIPC at initial diagnosis among Asians.

KEYWORDS: Epidermal growth factor receptor mutation, epidermal growth factor receptor tyrosine kinase inhibitor, gefitinib, lung cancer, miliary carcinomatosis

ung cancer is the leading cause of cancerrelated deaths, accounting for $28 \%$ of all cancer deaths in the USA and $>1$ million deaths worldwide annually [1, 2]. Although imaging techniques have advanced, $\sim 70 \%$ of nonsmall cell lung cancer (NSCLC) patients are still not diagnosed until the advanced stages of the disease. Their prognoses are also poor $[2,3]$.

The lung is frequently a metastatic organ of NSCLC, and lung metastasis presents with several different patterns on chest radiography, including multiple pulmonary nodules, pleural effusions and enlarged lymph nodes [4-7]. However, lung cancer with miliary intrapulmonary carcinomatosis (MIPC) is an uncommon phenomenon. A miliary pattern is defined as having diffuse, tiny and discrete pulmonary micronodules of $\leqslant 5 \mathrm{~mm}$ on chest radiography [7], indicating haematogenous dissemination [5, 8]. High-resolution computed tomography $(\mathrm{CT})$ provides better and more accurate detection of the widespread micronodules $[5,7]$.

NSCLC patients who present with MIPC at initial diagnosis have rapidly fatal courses although some case reports have described patients having a good response to epidermal growth factor receptor (EGFR) tyrosine kinase inhibitor (TKI) therapy [9, 10]. EGFR controls cell proliferation, differentiation and invasion [11]. It has been found that females, nonsmokers, those with adenocarcinoma and East Asians exhibit increased responses to EGFR-TKI treatment [12, 13], which is associated with increased EGFR mutation rates [14-19]. The L858R mutation and deletion in exon-19 (del-19) account for $90 \%$ of the EGFR mutations [15, 17, 18, 20].

LAACK et al. [21] reported five never-smokers with miliary pattern lung adenocarcinoma all having
AFFILIATIONS

*Dept of Internal Medicine, National Taiwan University Hospital Yun-Lin Branch, Yun-Lin,

*International Harvard Statistical Consulting Company,

'Dept of Pathology, National Taiwan University Hospital,

${ }^{+}$Dept of Surgery, National Taiwan University Hospital,

${ }^{\S}$ Dept of Internal Medicine, National Taiwan University Hospital,

**Dept of Medical Imaging, Nationa Taiwan University Hospital, and ${ }^{f}$ College of Medicine, National Taiwan University, Taipei, Taiwan.

CORRESPONDENCE

J-Y. Shih

Dept of Internal Medicine National Taiwan University Hospital No. 7 Chung-Shan South Road Taipei 100

Taiwan

E-mail: jyshih@ntu.edu.tw

Received:

Jan 112012

Accepted after revision:

March 272012

First published online:

April 202012 
del-19 EGFR mutations. They had good response to EGFRTKIs. However, the clinical characteristics and treatment outcomes of NSCLC patients presenting with MIPC were not described.

This study investigates the clinical characteristics, EGFR mutation rate and treatment response, and explores prognostic factors for NSCLC patients who presented with MIPC at initial diagnosis.

\section{MATERIALS AND METHODS}

\section{Patients}

All NSCLC patients diagnosed from June 2004 to December 2008 were identified through the Cancer Registry at the Medical Information Management Office of National Taiwan University Hospital (NTUH) (Taipei, Taiwan). Patients with MIPC at initial diagnosis were enrolled. This study was approved by the NTUH Research Ethics Committee (approval number 9561705036).

For comparison, the EGFR mutation status of patients with stage IV lung cancer without MIPC was identified. Patients screened for EGFR mutations were: 1) those who underwent fine-needle biopsies (CT/ultrasound-guided) or thoracentesis for pleural effusions after July 2004, when consecutive recruitment for EGFR mutations test was started at the hospital [22]; or 2) those who were included for retrospective NSCLC studies $[19,23-25]$.

\section{MIPC image-based criteria}

The MIPC image-based criteria were: 1) profuse, tiny, discrete and round pulmonary nodules that are generally uniform in size and diffusely distributed throughout both lung fields; 2) number of nodules not easily counted by CT; and 3) most of the pulmonary nodules were $\leqslant 5 \mathrm{~mm}$ in diameter (fig. $1 \mathrm{a}$ and b) $[7,26,27]$. Patients with unilateral intrapulmonary carcinomatosis, multifocal ground-glass opacities or lymphangitic carcinomatosis were excluded.

\section{Clinical data}

The clinical characteristics of eligible patients were recorded. Weight loss was defined as $>10 \%$ loss of the original body weight in 6 months. We defined patients who smoked $<100$ cigarettes in their lifetime as nonsmokers, patients who smoked cigarettes within a year of diagnosis as current smokers and the remaining patients as former smokers. Disease stage was determined by the Tumour Node Metastasis system [28].
Lung cancer histology was classified using the World Health Organization criteria [29]. Lung cancer was confirmed by pathological or cytological diagnoses using tissues obtained from biopsy or aspiration.

\section{Response evaluation of NSCLC patients who received first- line systemic treatment}

The timing and order of different treatment regimens was at the physicians' discretion after considering the patients clinical situation and a thorough discussion with patients. First-line systemic treatment for NSCLC patients included chemotherapy and EGFR-TKI therapy. The EGFR-TKIs gefitinib $250 \mathrm{mg} \cdot \mathrm{day}^{-1}$ (Iressa ${ }^{*}$; AstraZeneca, Wilmington, DE, USA) or erlotinib $150 \mathrm{mg} \cdot \mathrm{day}^{-1}$ (Tarceva ${ }^{\circledR}$; OSI Pharmaceuticals, Inc., Melville, NY, USA) were prescribed. Patients who received no systemic therapy received best supportive care. To evaluate treatment response, a chest radiograph was obtained every $2-4$ weeks and a chest CT every 2-3 months.

All images were reviewed by a pulmonologist (S-G. Wu) and a radiologist (Y-C. Chang) who were blinded to the EGFR mutation analysis results and treatment course of the study subjects. Treatment response was evaluated using the Response Evaluation Criteria in Solid Tumour guidelines (version 1.1) [30]. For measurable lesions, the responses were classified into complete response, partial response, stable disease or progressive disease. For patients who had only miliary lesions that were not measurable but evaluable, treatment responses were classified as nonprogressive disease (NPD) or progressive disease. NPD was defined as no new lesions and no unequivocal progression of existing lesions [30]. Disease control rate was calculated from all patients who had complete response, partial response, stable disease or NPD.

Overall survival was defined as the period from the date of NSCLC diagnosis to the date of death. Progression-free survival (PFS) was defined as the period from the date of first-line systemic treatment initiation to the date of the first objective or clinical sign of disease progression or death.

\section{Tissue procurement for EGFR mutation analysis}

Tumour specimens from lung tumours, metastatic sites and malignant effusion cell blocks were obtained for mutation analysis. Written informed consent to use tissue for molecular analysis was obtained from patients at the time of specimen collection. Tissue sections were examined for adequacy by microscopy with haematoxylin and eosin staining; tissue
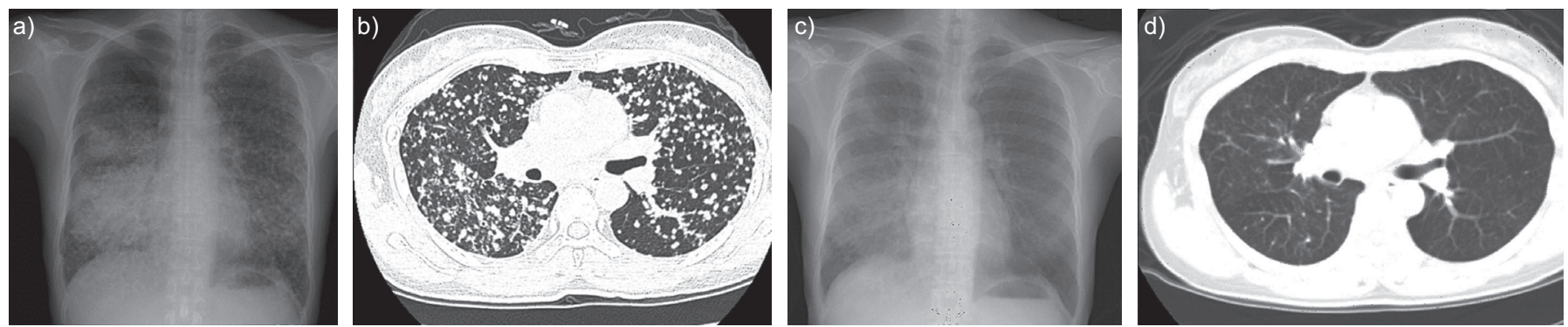

FIGURE 1. a, c) Chest radiography and b, d) computed tomography showing a patient a, b) with miliary intrapulmonary carcinomatosis at initial diagnosis. c, d) After treatment with the epidermal growth factor receptor tyrosine kinase inhibitor gefitinib for 2 months, the images showed an obvious decrease of the miliary lung metastases. 
samples that consisted of $>80 \%$ tumour content were selected for the study.

\section{PCR amplification and direct sequencing}

Tumour DNA, obtained from paraffin blocks using a QiAmp DNA Mini kit (Qiagen, Valencia, CA, USA), was used for EGFR mutation analysis as described previously [19, 23, 25]. The tyrosine kinase domain of the EGFR coding sequence (exons 18-21) was amplified by independent rounds of PCR. The PCR amplicons were purified and sequenced using the BigDye Terminator Sequencing Kit (Applied Biosystems, Foster City, CA, USA).

\section{Statistical analysis}

All analyses were performed using the statistical software SPSS 15.0 (SPSS Inc., Chicago, IL, USA) or SAS 9.1.3 (SAS Institute Inc., Cary, NC, USA). Two-sided p-values of $<0.05$ were considered statistically significant. All categorical variables were analysed by the Chi-squared test, except those with an expected frequency of less than five, which were analysed by Fisher's exact test. Unpaired t-tests were used for mean comparisons of continuous variables between two groups. Survival curves were plotted using the Kaplan-Meier method and compared between groups using the log-rank test.

To account for the patients without EGFR sequencing results, a logistic regression analysis was conducted on the predictive factors of the "missing" EGFR sequencing results versus "nonmissing" to estimate the probability of "missing". Further regression analysis with EGFR mutation as a covariate was weighted by the inverse of the predicted probability of "nonmissing" from the fitted logistic regression model for "missing" to obtain valid result. A weighted Cox's proportional hazards model was used to identify predictive factors of overall survival in the nonmissing patients with MIPC [31, 32].

\section{RESULTS}

\section{Clinical characteristics of patients presenting with MIPC at} initial diagnosis

From June 2004 to December 2008, there were 3,612 NSCLC patients registered in the Cancer Registry. Among them, 85 ( $2 \%)$ patients presented with MIPC at initial diagnosis. Of the 85 patients, 41 (48\%) were male. The mean age was 59.8 yrs (range 28.3-87.7 yrs). There were 63 (74\%) nonsmokers. 81 (95\%) patients had adenocarcinoma and one had squamous cell carcinoma. The remaining three patients had NSCLC not otherwise specified. The most frequent distant metastasis sites were bone $(64 \%)$ and brain $(37 \%)$. Other clinical characteristics are shown in table 1.

The most common respiratory and nonrespiratory symptoms were coughing $(66 \%)$ and weight loss $(45 \%)$, respectively. Other symptoms are listed in table S1.

\section{EGFR mutation status of patients presenting with MIPC at initial diagnosis}

EGFR mutation testing was performed for 60 (71\%) patients with both informed consent and adequate tissue samples. The specimens were obtained from lung tumours $(n=23)$, pleural effusions $(n=28)$, cervical lymph nodes $(n=4)$, metastatic brain lesions $(n=3)$, a metastatic bony lesion $(n=1)$ and ascites $(n=1)$. Patient demographics are shown in table 1.
There were $42(70 \%)$ patients with EGFR mutations. The mutation rates did not differ significantly by $\operatorname{sex}(p=0.052)$ or smoking status $(\mathrm{p}=0.194)$ (table 2$)$. It was noted that mutation rates for males and smokers were $84 \%$ (21 out of 25 ) and $85 \%$ (11 out of 13), respectively. The most common mutations were del-19 $(n=21,35 \%)$ and L858R $(n=12,20 \%)($ table S2).

\section{Characteristics and EGFR mutation status of stage IV lung cancer patients without MIPC at initial diagnosis}

From April 2001 to November 2009, 673 patients with stage IV lung cancer without MIPC were identified for EGFR mutation screening. Compared to patients with MIPC and EGFR mutation status, there was no difference in sex, age, smoking history or tumour type between the two groups. The patients with MIPC had higher metastasis rates of bone $(p=0.022)$, brain $(\mathrm{p}=0.021)$ and liver $(\mathrm{p}=0.010)($ table 3$)$.

Patients who presented with MIPC at initial diagnosis had a higher EGFR mutation rate than patients without MIPC $(70 \%$ versus $56 \%, p=0.036$ ) (table 4 ). The presence of L858R mutation did not differ significantly between the two groups $(20 \%$ versus $22 \%, \mathrm{p}=0.433$ ). However, patients with MIPC had significantly higher rate of del-19 mutation ( $35 \%$ versus $25 \%, \mathrm{p}=0.024)$ (table 4).

\begin{tabular}{|c|c|c|c|}
\hline \multirow{3}{*}{ TABLE 1} & \multirow{2}{*}{\multicolumn{3}{|c|}{$\begin{array}{l}\text { Clinical characteristics of nonsmall cell lung } \\
\text { cancer patients who presented with miliary } \\
\text { intrapulmonary carcinomatosis at initial } \\
\text { diagnosis }\end{array}$}} \\
\hline & & & \\
\hline & Patients & EGFR tested & p-value ${ }^{\#}$ \\
\hline Total $\mathbf{n}$ & 85 & 60 & \\
\hline Age yrs & $59.8(28.3-87.7)$ & $61.4(39.1-87.7)$ & $0.070^{+}$ \\
\hline Sex & & & 0.060 \\
\hline Females & $44(52)$ & $35(58)$ & \\
\hline Males & $41(48)$ & $25(42)$ & \\
\hline Smoking & & & 0.169 \\
\hline Nonsmokers & $63(74)$ & $47(78)$ & \\
\hline Former/current smokers & $22(26)$ & $13(22)$ & \\
\hline ECOG PS & & & 0.055 \\
\hline $0-1$ & $63(74)$ & $48(80)$ & \\
\hline $2-4$ & $22(26)$ & $12(20)$ & \\
\hline Tumour type & & & $0.577^{\S}$ \\
\hline Nonadenocarcinoma & $4(5)$ & $2(3)$ & \\
\hline Adenocarcinoma & $81(95)$ & $58(97)$ & \\
\hline \multicolumn{4}{|l|}{ Distant metastasis } \\
\hline Bone & $54(64)$ & $37(62)$ & 0.580 \\
\hline Brain & $31(37)$ & $22(37)$ & 0.954 \\
\hline Liver & $23(27)$ & $17(28)$ & 0.682 \\
\hline Adrenal gland & $8(9)$ & $8(13)$ & $0.098^{\S}$ \\
\hline Others" & $12(14)$ & $7(12)$ & 0.315 \\
\hline
\end{tabular}

Data are presented as $\mathrm{n}(\%)$ or mean (range), unless otherwise stated. EGFR epidermal growth factor receptor; ECOG PS: Eastern Cooperative Oncology Group performance status. ${ }^{\#}$ : comparison between the groups with and without EGFR sequencing; ': five spleen, one peritoneal, two cervical lymph nodes, three intra-abdominal lymphoadenopathy and one kidney; ${ }^{+}$: t-test. ${ }^{\text {s. }}$. Fisher's exact test. 


\begin{tabular}{|c|c|c|c|c|}
\hline \multirow[t]{2}{*}{ TABLE 2} & \multicolumn{4}{|c|}{$\begin{array}{l}\text { EGFR mutation status of nonsmall cell lung } \\
\text { cancer patients who presented with miliary } \\
\text { intrapulmonary carcinomatosis at initial } \\
\text { diagnosis }\end{array}$} \\
\hline & & EGFR mutation & Wild type & p-value \\
\hline \multicolumn{2}{|l|}{ Total } & 42 & 18 & \\
\hline \multicolumn{2}{|l|}{ Age yrs } & $61.2(41.3-87.7)$ & $61.7(39.1-79.6)$ & $0.889^{\circ}$ \\
\hline \multicolumn{2}{|l|}{ Sex } & & & $0.052^{+}$ \\
\hline \multicolumn{2}{|l|}{ Females } & 21 & 14 & \\
\hline \multicolumn{2}{|l|}{ Males } & 21 & 4 & \\
\hline \multicolumn{2}{|l|}{ Smoking } & & & $0.308^{+}$ \\
\hline \multicolumn{2}{|c|}{ Nonsmokers } & 31 & 16 & \\
\hline \multicolumn{2}{|c|}{ Former/current smokers } & 11 & 2 & \\
\hline \multicolumn{2}{|c|}{ ECOG PS } & & & $1.000^{+}$ \\
\hline \multicolumn{2}{|l|}{$0-1$} & 33 & 15 & \\
\hline \multicolumn{2}{|l|}{$2-4$} & 9 & 3 & \\
\hline \multicolumn{2}{|l|}{ Tumour type } & & & $0.514^{+}$ \\
\hline \multicolumn{2}{|c|}{ Nonadenocarcinoma } & 1 & 1 & \\
\hline \multicolumn{2}{|c|}{ Adenocarcinoma } & 41 & 17 & \\
\hline \multicolumn{5}{|c|}{ Distant metastasis } \\
\hline \multicolumn{2}{|l|}{ Bone } & 28 & 9 & \\
\hline \multicolumn{2}{|l|}{ Brain } & 17 & 5 & \\
\hline \multicolumn{2}{|l|}{ Liver } & 12 & 5 & \\
\hline \multicolumn{2}{|c|}{ Adrenal gland } & 6 & 2 & \\
\hline \multicolumn{2}{|l|}{ Others ${ }^{\#}$} & 5 & 2 & \\
\hline
\end{tabular}

Data are presented as $\mathrm{n}$ or mean (range), unless otherwise stated. EGFR: epidermal growth factor receptor; ECOG PS: Eastern Cooperative Oncology Group performance status. \#: three spleen, one peritoneal, one cervical lymph node and two intra-abdominal lymphadenopathies; " $"$ :t-test; ${ }^{+}$: Fisher's exact test.

\section{Response rate and PFS after first-line systemic treatment}

Of the 85 patients with MIPC at initial diagnosis, 80 received systemic treatment. Three patients were grade 4 on the Eastern Cooperative Oncology Group performance status scale at diagnosis, whose clinical conditions worsened rapidly. Two patients refused systemic treatment. The treatment selection depended on the physicians' discretion. The distributions of treatment sequences and their EGFR mutation status of patients were shown in table S3.

First-line treatments were EGFR-TKI for 43 patients and chemotherapy for 37 patients. In the EGFR-TKI group, 33 received gefitinib and 10 received erlotinib. Among them, 29 had a partial response (fig. 1c and d), one had NPD and 13 exhibited progressive disease; the disease control rate was $70 \%$. In the chemotherapy group, first-line chemotherapies included platinum-based doublet chemotherapy $(n=29)$, gemcitabine $(n=4)$, vinorelbine $(n=3)$ and paclitaxel $(n=1)$. None received bevacizumab as combination chemotherapy. In this group, 11 patients had a partial response, four patients had NPD and 22 patients had progressive disease; the disease control rate was $41 \%$. Disease control rates between the two groups were different $(p=0.009)$. Furthermore, the EGFR-TKI group had longer median PFS (5.8 versus 2.9 months, $\mathrm{p}=0.001$ ) (fig. 2).

\begin{tabular}{|c|c|c|c|c|}
\hline \multirow[t]{2}{*}{ TABLE 3} & \multicolumn{4}{|c|}{$\begin{array}{l}\text { Clinical characteristics of stage IV nonsmall cell } \\
\text { lung cancer patients who presented with and } \\
\text { without miliary intrapulmonary carcinomatosis } \\
\text { (MIPC) at initial diagnosis }\end{array}$} \\
\hline & & With MIPC & Without MIPC & $p$-value \\
\hline \multicolumn{2}{|l|}{ Total } & 60 & 673 & \\
\hline \multicolumn{2}{|l|}{ Age yrs } & $61.4(39.1-87.7)$ & $63.9(24.8-91.4)$ & $0.130^{\#}$ \\
\hline \multicolumn{2}{|l|}{ Sex } & & & 0.485 \\
\hline \multicolumn{2}{|l|}{ Females } & 35 & 361 & \\
\hline \multicolumn{2}{|l|}{ Males } & 25 & 312 & \\
\hline \multicolumn{2}{|l|}{ Smoking } & & & 0.091 \\
\hline \multicolumn{2}{|l|}{ Nonsmokers } & 47 & 456 & \\
\hline \multicolumn{2}{|c|}{ Former/current smokers } & 13 & 217 & \\
\hline \multicolumn{2}{|l|}{ Tumour type } & & & $1.000^{\circ}$ \\
\hline \multicolumn{2}{|c|}{ Nonadenocarcinoma } & 2 & 33 & \\
\hline \multicolumn{2}{|c|}{ Adenocarcinoma } & 58 & 640 & \\
\hline \multicolumn{5}{|c|}{ Distant metastasis } \\
\hline \multicolumn{2}{|l|}{ Bone } & 37 & 311 & 0.022 \\
\hline \multicolumn{2}{|l|}{ Brain } & 22 & 157 & 0.021 \\
\hline \multicolumn{2}{|l|}{ Liver } & 17 & 104 & 0.010 \\
\hline \multicolumn{2}{|c|}{ Malignant pleural effusion } & 27 & 331 & 0.535 \\
\hline \multicolumn{2}{|c|}{ Adrenal gland } & 8 & 74 & 0.582 \\
\hline \multicolumn{2}{|l|}{ Others } & 7 & 71 & 0.788 \\
\hline
\end{tabular}

Data are presented as $\mathrm{n}$ or mean (range), unless otherwise stated. $\mathrm{p}$-values were calculated by the Chi-squared test, unless otherwise stated. " ${ }^{*}$ : t-test; " Fisher's exact test.

\section{Overall survival and prognostic factors}

Univariate analysis of prognostic factors of the 85 patients with MIPC is shown in table 5 . The difference in overall survival between patients with adenocarcinoma and nonadenocarcinoma was statistically significant $(p=0.049)$. Patients without extrapulmonary metastasis had longer overall survival than those with extrapulmonary metastasis $(\mathrm{p}=0.032)$ (fig. 3). Patients who received EGFR-TKI sometime during their illness had significantly longer overall survival than those who did not $(\mathrm{p}<0.001)$.

\begin{tabular}{llllll} 
TABLE 4 & $\begin{array}{l}\text { The difference in EGFR mutation types between } \\
\text { stage IV nonsmall cell lung cancer with and } \\
\text { without miliary intrapulmonary carcinomatosis } \\
\text { (MIPC) at initial diagnosis }\end{array}$ \\
& Wild type & L858R & del-19 & Other & Total \\
\hline $\begin{array}{l}\text { With MIPC } \\
\text { Without MIPC }\end{array}$ & $296(44)$ & $149(22)$ & $165(25)$ & $63(9)$ & 673 \\
\hline
\end{tabular}

Data are presented as $n(\%)$ or $n . p=0.036$ for MIPC versus non-MIPC stage IV nonsmall cell lung cancer specimens for positive and negative of epidermal growth factor receptor (EGFR) mutations; $\mathrm{p}=0.024$ for the patient presented with versus without MIPC at initial diagnosis with del-19 mutations. del-19: inframe deletion in exon-19. 


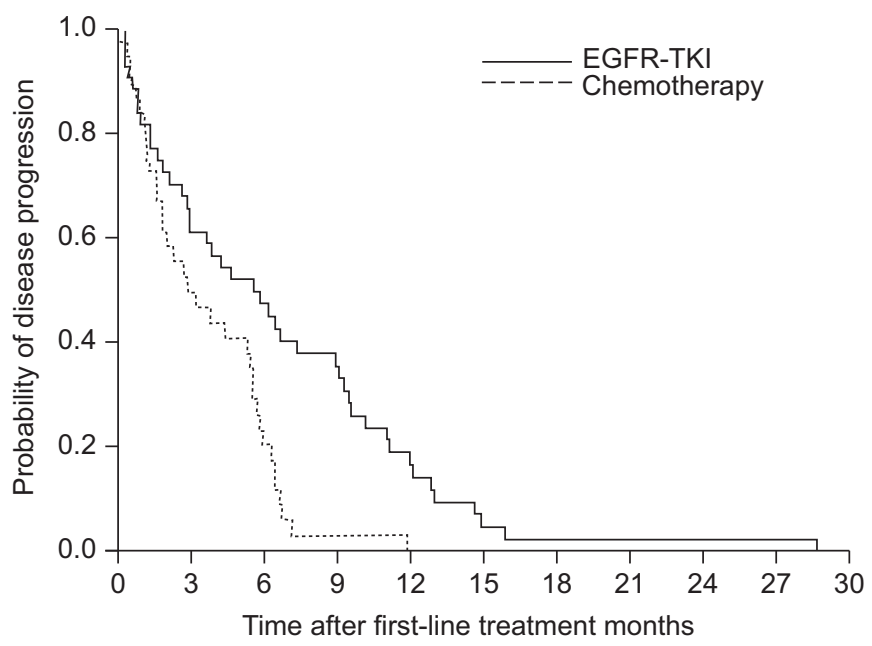

FIGURE 2. Kaplan-Meier curve of progression-free survival (PFS) in nonsmall cell lung cancer patients with miliary intrapulmonary carcinomatosis at initial diagnosis who received epidermal growth factor receptor (EGFR) tyrosine kinase inhibitor (TKI) $(n=43)$ and systemic chemotherapy $(n=37)$ as first-line treatment. The difference in PFS was statistically significant (median 5.8 versus 2.9 months, $p=0.001$ by the log-rank test).

Among the 60 patients with a known EGFR sequence, 55 received EGFR-TKI treatment; among them, 38 (69\%) had EGFR mutations. Of those treated with EGFR-TKIs, 35 were treated as the first-line treatment and 21 as the second- or subsequent-line treatment. Patients with EGFR mutations had longer PFS than those with wild-type EGFR (9.2 versus 2.7 months, $\mathrm{p}<0.001$ ) (fig. S1). The difference in overall survival between patients with and without EGFR mutations was statistically significant (17.8 versus 10.6 months, $\mathrm{p}=0.008$ ) (fig. 4 ).

Overall survival analysis based on the order of treatment received showed that patients who received EGFR-TKI therapy as firstline treatment and chemotherapy as second-line treatment had the longest overall survival (median 20.9 months). This is followed sequentially by patients who received chemotherapy as both first- and second-line treatments, chemotherapy with subsequent EGFR-TKI, EGFR-TKI only, chemotherapy only and, lastly, supportive care alone $(\mathrm{p}<0.001)$ (table 5).

Logistic regression of the 25 patients with no EGFR sequencing showed that they were primarily male $(p=0.052)$, had poorer performance status $(p=0.035)$ and received chemotherapy as the first-line treatment $(p=0.006)$. Their EGFR status was weighted according to these factors. Multivariate analysis of overall survival was then performed using the Cox proportional hazard model for potential prognostic factors, including sex, age, smoking status, tumour type, extrapulmonary metastasis, EGFR mutation status, EGFR-TKI use and treatment order.

Longer overall survival was associated with having adenocarcinoma (hazard ratio (HR) 0.16, $\mathrm{p}=0.0448$ ), absence of extrapulmonary metastasis (HR 0.45, $\mathrm{p}=0.0514)$ and EGFR mutation (HR 0.19, $\mathrm{p}=0.0001$ ). Factors with a significantly negative effect on overall survival were best supportive care (HR 106.19, $\mathrm{p}<0.0001)$ and the use of first-line EGFR-TKI therapy as the only treatment (HR 4.46, $\mathrm{p}=0.0003)$ (table 5). The measured goodness-of-fit values and the results of the goodness-of-fit test indicated that the Cox proportional hazards model fitted the observed binary data well.

\section{DISCUSSION}

NSCLC patients with EGFR mutations are known to have better response to EGFR-TKI treatment [14, 16]. The present study showed that NSCLC patients presented with MIPC at initial diagnosis had a higher EGFR mutation rate than those without MIPC. We enrolled more patients than the five cases originally reported by LAACK et al. [21]; therefore, we are able to present the complete clinical characteristics, metastatic sites and EGFR mutation types in this subgroup of patients. Currently known clinical characteristics associated with EGFR mutation include female sex, adenocarcinoma, never having smoked and being Asian $[17,33]$. Our finding of a high EGFR mutation rate among patients presenting with the special image pattern of MIPC at initial diagnosis provide physicians an additional characteristic in selecting patients who may have better response to EGFR-TKIs.

The Iressa Pan-Asia Study (IPASS), conducted on East Asian nonsmokers and former light smokers with lung adenocarcinoma, showed that initial gefitinib treatment resulted in a better response rate and longer PFS than carboplatin-paclitaxel treatment [16]. The EGFR mutation rate of IPASS was $60 \%$, which is lower than the $70 \%$ found in the present study. Furthermore, contrary to the general concept regarding high EGFR mutation subgroups [12, 13], we also found that the subgroups of NSCLC patients with MIPC, males and smokers, have high EGFR mutation rates. The high EGFR mutation rate was consistent with a better treatment response and longer PFS among patients treated with EGFR-TKI as first-line treatment.

MIPC at initial diagnosis is a more invasive disease state. Most NSCLC patients with MIPC at initial diagnosis often have poor performance status and rapidly fatal courses [34], and chemotherapy is not usually recommended [35]. The present study showed a high EGFR mutation rate in this subgroup of NSCLC patients. In addition, best supportive care had a very short overall survival. EGFR-TKIs may be the treatment of choice for NSCLC patients with MIPC, especially for patients with poor performance status.

The mutation rate of del-19 was higher in patients with MIPC than those without MIPC (35\% versus $25 \%$ ). The high del-19 mutation rate resulted in a higher EGFR mutation rate in patients with MIPC than those without MIPC at initial diagnosis. Further studies are necessary to investigate the relationship between del-19 mutation and miliary pulmonary metastasis in NSCLC.

Tumour cell metastasis via the haematogenous route can result in diffuse miliary seeding [36]. UMEKI et al. [34] showed that MIPC of lung cancer was associated with bone metastasis because all five of his patients had bone metastases. They proposed that bone metastasis occurred from the lung via the haematogenous route, whereas MIPC arises from multiple tumour emboli from secondary bone metastatic foci [34]. Kolsuz et al. [37] described a lung adenocarcinoma patient with MIPC and bone marrow involvement, which was thought to be caused by tumour cell spread via the haematogenous 


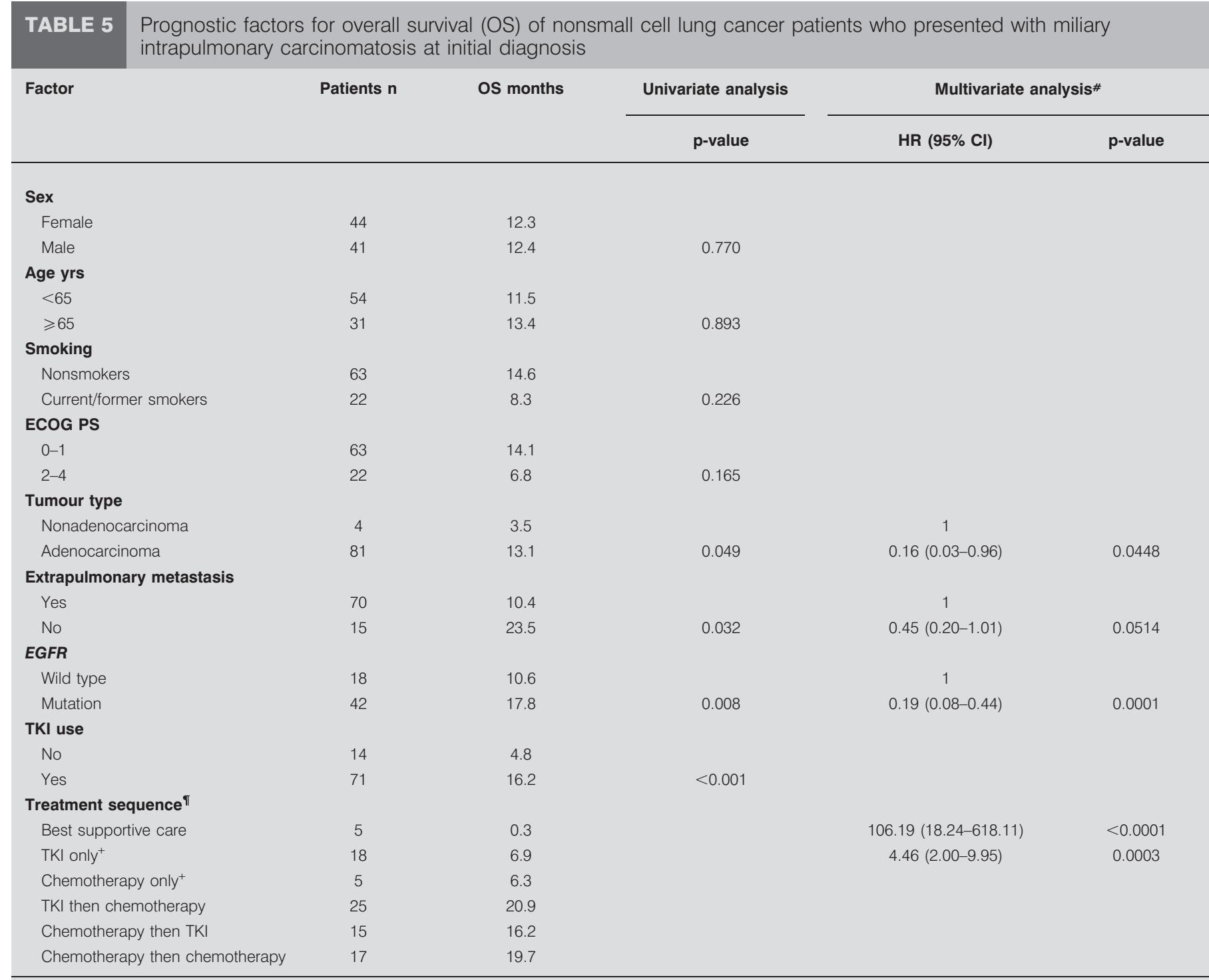

HR: hazard ratio; ECOG PS: Eastern Cooperative Oncology Group performance status; EGFR: epidermal growth factor receptor, TKI: tyrosine kinase inhibitor. ${ }^{*}$ : before performing multivariate analysis, we performed logistic regression to identify potential factors associated with the 25 patients lacking EGFR sequencing data. Potential factors mainly included male sex, poor performance status and chemotherapy as the first-line treatment. After weighting the missing data of the 25 patients lacking EGFR sequencing data by logistic regression, we used the Cox proportional hazard method for multivariate analysis of OS. " : the sequence of patient treatment courses was classified according to the first- and second-line medications. ${ }^{+}$: TKI-only and chemotherapy-only patients received only first-line systemic treatment without second-line treatment.

route. The present study also showed that $63.5 \%$ of NSCLC patients with MIPC at initial diagnosis had synchronous bone metastasis (table 1). In comparison with the stage IV NSCLC patients presented without MIPC at initial diagnosis, patients with MIPC had higher rates of metastasis to the liver, bone and brain, all sites of haematogenous spread. This result supports that haematogenous spread plays an important role in both MIPC and bone metastases of NSCLC.

The present study showed that the majority of NSCLC patients with MIPC at initial diagnosis had adenocarcinoma. The patients with MIPC in the previous reports all had adenocarcinoma [21,34]. The probable pathophysiology is that miliary presentation is a manifestation of haematogenous spread, which is associated with angiogenesis of cancer [5]. In comparison with lung squamous cell carcinoma, lung adenocarcinoma has more tumour angiogenic potential, which may contribute to the high haematogenous spread [38]. In addition, lung adenocarcinoma may develop early metastasis via haematogenous spread $[5,8,39]$. Therefore, adenocarcinoma is the dominant histopathology type of MIPC at initial diagnosis.

Despite multiple pulmonary metastases, patients may still respond to EGFR-TKIs. CHANG et al. [10] reported successful treatment of multifocal bronchioloalveolar cell carcinoma with gefitinib in two patients. GOTO et al. [40] showed that NSCLC patients who had more than six metastatic pulmonary nodules (particularly diffuse miliary metastases) were significantly 


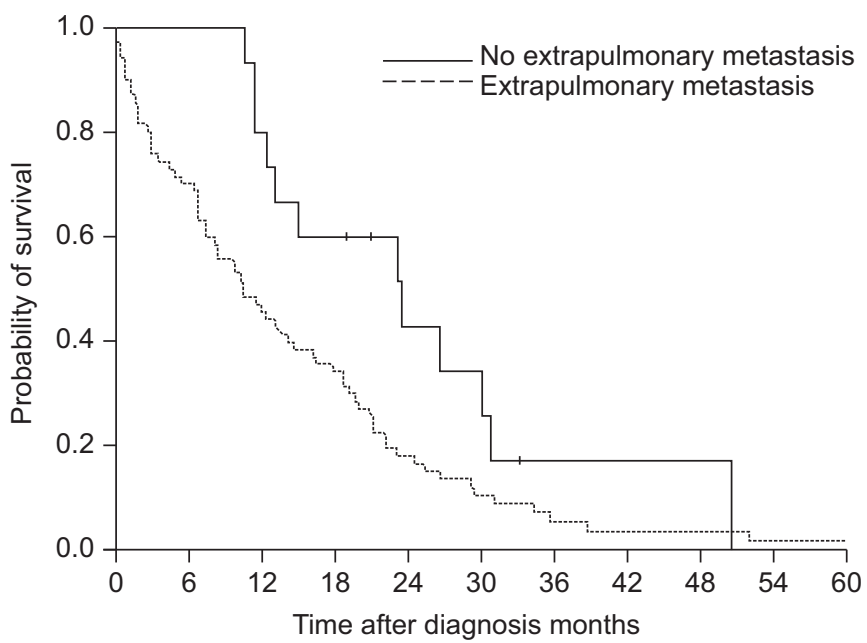

FIGURE 3. Kaplan-Meier curve of overall survival in nonsmall cell lung cancer patients with miliary intrapulmonary carcinomatosis at initial diagnosis. The difference in overall survival between patients without extrapulmonary metastasis $(n=15)$ and those with extrapulmonary metastasis $(n=70)$ was statistically significant (median 23.5 versus 10.4 months, $p=0.032$ by the log-rank test).

associated with a positive response to gefitinib. However, EGFR mutation status, the most important factor in determining response to EGFR-TKI treatment, was not known at that time. In 2006, KOBAYASHI et al. [9] linked EGFR mutation with a good gefitinib response among two diffuse micronodular pulmonary metastasis patients. In our study, we also showed that patients presenting with MIPC responded well to EGFRTKIs, which may be associated with the high EGFR mutation rate among our patients.

The present study has some limitations. First, the patient number was small because MIPC is a rare presentation of lung

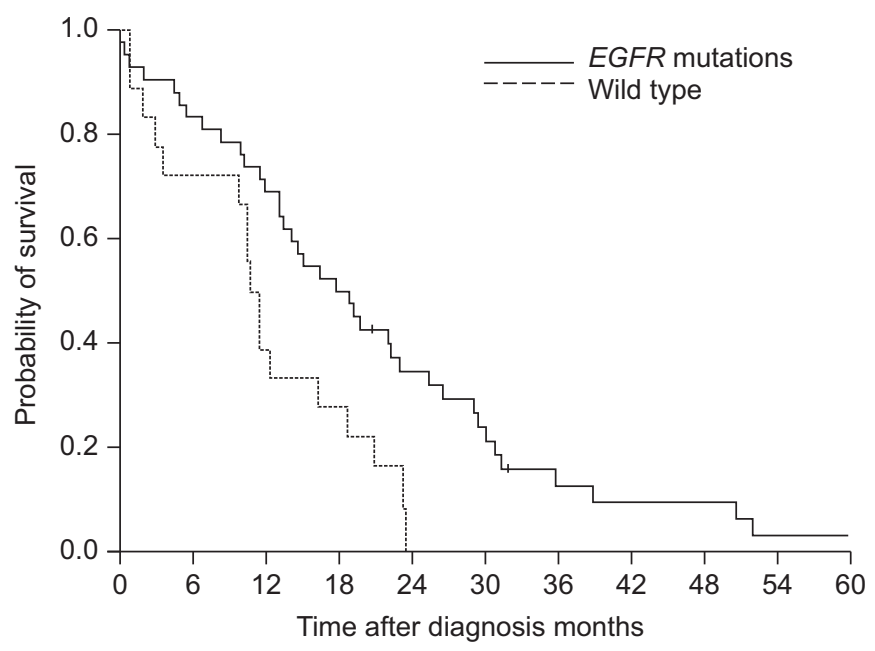

FIGURE 4. Kaplan-Meier curve of overall survival after first-line treatment in non-small cell lung cancer patients with miliary intrapulmonary carcinomatosis at initial diagnosis. The difference in overall survival between the patients with epidermal growth factor receptor (EGFR) mutations $(\mathrm{n}=42)$ and those with wild-type EGFR ( $\mathrm{n}=18$ ) was statistically significant (median 17.8 versus 10.6 months, $p=0.008$ by the log-rank test). cancer at diagnosis. Secondly, this was a retrospective observational study, and inherent biases cannot be completely excluded. Thirdly, differentiating intrapulmonary metastasis from independent multiple primary tumours is difficult in miliary pulmonary nodules of NSCLC. Comprehensive histological assessment and molecular analysis of different nodules are powerful tools [41], but obtaining specimens from multiple micronodules is difficult because the lesions are too small. Lastly, all enrolled patients in this study were Asian, known to have higher EGFR mutation rate. The results may not be generalisable to all patients presenting with MIPC.

In conclusion, NSCLC patients presenting with MIPC at initial diagnosis have high rates of adenocarcinoma and EGFR mutation rate even among males and smokers. MIPC pattern helps to identify patients who have a high likelihood of having EGFR mutation. EGFR-TKI may be the treatment of choice for NSCLC patients with MIPC at initial diagnosis among Asians regardless of sex or smoking status.

\section{SUPPORT STATEMENT}

This study was supported by grants $98-2314-\mathrm{B}-002-117-\mathrm{MY} 3$ and 982628-B-002-087-MY3 (National Science Council, Taipei, Taiwan), grant DOH100-TD-PB-111-TM001 (Dept of Health, Executive Yuan, Taipei), and grant 100-M1722 (National Taiwan University Hospital, Taipei).

\section{STATEMENT OF INTEREST}

None declared.

\section{ACKNOWLEDGEMENTS}

The authors would like to thank the Dept of Medical Research, National Taiwan University Hospital (Taipei, Taiwan) for technical support.

\section{REFERENCES}

1 Parkin DM, Bray F, Ferlay J, et al. Global cancer statistics, 2002. CA Cancer J Clin 2005; 55: 74-108.

2 Siegel R, Ward E, Brawley O, et al. Cancer statistics, 2011: the impact of eliminating socioeconomic and racial disparities on premature cancer deaths. CA Cancer J Clin 2011; 61: 212-236.

3 Woodward RM, Brown ML, Stewart ST, et al. The value of medical interventions for lung cancer in the elderly: results from SEERCMHSF. Cancer 2007; 110: 2511-2518.

4 Marom EM, Patz EF Jr, Swensen SJ. Radiologic findings of bronchogenic carcinoma with pulmonary metastases at presentation. Clin Radiol 1999; 54: 665-668.

5 Hansell DM, Bankier AA, MacMahon H, et al. Fleischner Society: glossary of terms for thoracic imaging. Radiology 2008; 246: 697-722.

6 Quinn D, Gianlupi A, Broste S. The changing radiographic presentation of bronchogenic carcinoma with reference to cell types. Chest 1996; 110: 1474-1479.

7 Andreu J, Mauleon S, Pallisa E, et al. Miliary lung disease revisited. Curr Probl Diagn Radiol 2002; 31: 189-197.

8 Zompatori M, Bna C, Poletti V, et al. Diagnostic imaging of diffuse infiltrative disease of the lung. Respiration 2004; 71: 4-19.

9 Kobayashi M, Takeuchi T, Bandobashi K, et al. Diffuse micronodular pulmonary metastasis of lung adenocarcinoma predicts gefitinib response in association with epidermal growth factor receptor mutations. Anticancer Res 2006; 26: 1621-1626.

10 Chang GC, Yang TY, Wang NS, et al. Successful treatment of multifocal bronchioloalveolar cell carcinoma with ZD1839 (Iressa) in two patients. J Formos Med Assoc 2003; 102: 407-411.

11 Davies RL, Grosse VA, Kucherlapati R, et al. Genetic analysis of epidermal growth factor action: assignment of human epidermal 
growth factor receptor gene to chromosome 7. Proc Natl Acad Sci USA 1980; 77: 4188-4192.

12 Kris MG, Natale RB, Herbst RS, et al. Efficacy of gefitinib, an inhibitor of the epidermal growth factor receptor tyrosine kinase, in symptomatic patients with non-small cell lung cancer: a randomized trial. JAMA 2003; 290: 2149-2158.

13 Fukuoka M, Yano S, Giaccone G, et al. Multi-institutional randomized phase II trial of gefitinib for previously treated patients with advanced non-small-cell lung cancer (The IDEAL 1 Trial) [corrected]. J Clin Oncol 2003; 21: 2237-2246.

14 Lynch TJ, Bell DW, Sordella R, et al. Activating mutations in the epidermal growth factor receptor underlying responsiveness of nonsmall-cell lung cancer to gefitinib. N Engl J Med 2004; 350: 2129-2139.

15 Paez JG, Janne PA, Lee JC, et al. EGFR mutations in lung cancer: correlation with clinical response to gefitinib therapy. Science 2004; 304: 1497-1500.

16 Mok TS, Wu YL, Thongprasert S, et al. Gefitinib or carboplatinpaclitaxel in pulmonary adenocarcinoma. N Engl J Med 2009; 361: 947-957.

17 Shigematsu H, Lin L, Takahashi T, et al. Clinical and biological features associated with epidermal growth factor receptor gene mutations in lung cancers. J Natl Cancer Inst 2005; 97: 339-346.

18 Rosell R, Moran T, Queralt C, et al. Screening for epidermal growth factor receptor mutations in lung cancer. N Engl J Med 2009; 361: 958-967.

19 Shih JY, Gow CH, Yu CJ, et al. Epidermal growth factor receptor mutations in needle biopsy/aspiration samples predict response to gefitinib therapy and survival of patients with advanced nonsmall cell lung cancer. Int J Cancer 2006; 118: 963-969.

20 Sharma SV, Bell DW, Settleman J, et al. Epidermal growth factor receptor mutations in lung cancer. Nat Rev Cancer 2007; 7: 169-181.

21 Laack E, Simon R, Regier $M$, et al. Miliary never-smoking adenocarcinoma of the lung: strong association with epidermal growth factor receptor exon 19 deletion. J Thorac Oncol 2011; 6: 199-202.

22 Wu SG, Gow CH, Yu CJ, et al. Frequent epidermal growth factor receptor gene mutations in malignant pleural effusion of lung adenocarcinoma. Eur Respir J 2008; 32: 924-930.

$23 \mathrm{Wu}$ JY, Yu CJ, Yang CH, et al. First- or second-line therapy with gefitinib produces equal survival in non-small cell lung cancer. Am J Respir Crit Care Med 2008; 178: 847-853.

$24 \mathrm{Wu}$ JY, Wu SG, Yang CH, et al. Lung cancer with epidermal growth factor receptor exon 20 mutations is associated with poor gefitinib treatment response. Clin Cancer Res 2008; 14: 4877-4882.

25 Gow $\mathrm{CH}$, Chang YL, Hsu YC, et al. Comparison of epidermal growth factor receptor mutations between primary and corresponding metastatic tumors in tyrosine kinase inhibitor-naive non-small-cell lung cancer. Ann Oncol 2009; 20: 696-702.
26 Rubins JB, Solomon R, Colby T, et al. Chest pain and progressive miliary infiltrates in an elderly man. Chest 2009; 135: 578-582.

27 Lee KS, Kim TS, Han J, et al. Diffuse micronodular lung disease: HRCT and pathologic findings. J Comput Assist Tomogr 1999; 23: 99-106.

28 Goldstraw P, Crowley J, Chansky K, et al. The IASLC Lung Cancer Staging Project: proposals for the revision of the TNM stage groupings in the forthcoming (seventh) edn of the TNM Classification of malignant tumours. J Thorac Oncol 2007; 2: 706-714.

29 Travis WDBE, Muller-Hermelink HK, Harris CC. Pathology and Genetics of Tumors of the Lung, Pleura, Thymus and Heart. Lyon, IARC Press, 2004.

30 Eisenhauer EA, Therasse P, Bogaerts J, et al. New response evaluation criteria in solid tumours: revised RECIST guideline (version 1.1). Eur J Cancer 2009; 45: 228-247.

31 Hosmer D, Lemeshow S. Applied Logistic Regression. 2nd Edn. New York, Wiley, 2000.

32 Kleinbaum D, Klein M. Logistic Regression: a Self-Learning Text. 2nd Edn. New York, Springer-Verlag, 2002.

33 Kosaka T, Yatabe Y, Endoh H, et al. Mutations of the epidermal growth factor receptor gene in lung cancer: biological and clinical implications. Cancer Res 2004; 64: 8919-8923.

34 Umeki S. Association of miliary lung metastases and bone metastases in bronchogenic carcinoma. Chest 1993; 104: 948-950.

35 Azzoli CG, Baker S Jr, Temin S, et al. American Society of Clinical Oncology Clinical Practice Guideline update on chemotherapy for stage IV non-small-cell lung cancer. J Clin Oncol 2009; 27: 6251-6266.

36 Coppage L, Shaw C, Curtis AM. Metastatic disease to the chest in patients with extrathoracic malignancy. J Thorac Imaging 1987; 2: 24-37.

37 Kolsuz M, Metintaş M, Dündar E, et al. A pulmonary adenocarcinoma with miliary lung metastases and bone marrow involvement. Turk Respir J 2000; 1: 45-47.

38 Yuan A, Yang PC, Yu CJ, et al. Tumor angiogenesis correlates with histologic type and metastasis in non-small-cell lung cancer. Am J Respir Crit Care Med 1995; 152: 2157-2162.

39 Travis WD, Travis LB, Devesa SS. Lung cancer. Cancer 1995; 75: 191-202.

40 Goto K, Kim E, Kubota K, et al. Association of multiple pulmonary metastases with response to gefitinib in patients with non-small cell lung cancer. J Clin Oncol 2004; 22: A7098.

41 Girard N, Deshpande C, Lau C, et al. Comprehensive histologic assessment helps to differentiate multiple lung primary nonsmall cell carcinomas from metastases. Am J Surg Pathol 2009; 33: $1752-1764$. 